\title{
"AHORA ME DA MIEDO SALIR SOLA, PORQUE ME DA MIEDO PERDERME": PERCEPCIÓN DE DESEMPEÑOY PARTICIPACIÓN EN ACTIVIDADES DE LA VIDA DIARIA EN UN GRUPO DE PERSONAS MAYORES CON DEMENCIA LEVE.
}

\author{
"NOW I'M AFRAID TO GO OUT ALONE, BECAUSE I'M AFRAID TO LOSING MYSELF": \\ PERFORMANCE AND PARTICIPATION IN DAILY LIFE ACTIVITIES PERCEPTION IN A \\ GROUP OF OLDER PERSONS WITH MILD DEMENTIA.
}

\section{José Aravena C. ${ }^{1}$, Diana Tello G. ${ }^{2}$ yTrinidad Callejas B. ${ }^{3}$}

\begin{abstract}
Resumen
Las personas con demencia leve pueden presentar diversas dificultades en la realización de sus actividades diarias, esto se debe a los síntomas clínicos que presentan, sin embargo la percepción que la persona tiene en relación a su propio desempeño también podría influir en la participación de sus actividades. Para conocer la percepción de las personas con demencia leve respecto a su propio desempeño en actividades cotidianas, se realizó un estudio cualitativo a través de grupos focales dirigido a 13 personas con demencia leve participantes del Centro Kintun. Los grupos focales fueron compuestos por cuatro a cinco personas donde se desarrollaron dos temáticas: las actividades importantes y las actividades donde perciben dificultades. A través de un análisis hermenéutico-interpretativo por medio de codificación abierta, se lograron identificar dos comportamientos en relación al desempeño diario de actividades: la necesidad de mantención de actividades y roles biográficamente significativos, y la auto-marginación de actividades o sensación de dependencia impuesta por el entorno social. Estos hallazgos resaltan la importancia de considerar la percepción de la persona con demencia y del entorno social en relación a las posibilidades de participación en actividades diarias de los sujetos, considerando que no sólo los síntomas clínicos pueden impactar en la participación sino que también la interpretación que se realiza de las situaciones cotidianas.
\end{abstract}

\section{Palabras clave:}

Demencia; Actividades de la vida diaria; Percepción; Desempeño de actividades.

\begin{abstract}
People with mild dementia may have many difficulties associated to clinical symptoms in daily life activities, but also the person perception about his daily performance might influence the participation in activities. To know the perception of the people with mild dementia about their performance in daily life activities, it was made a qualitative study, it was conducted through focus groups with 13 persons with dementia participants from Kintun Center. Groups of four to five persons were formed, where they discussed about two main topics: important activities and activities where they perceive difficulties. Through a hermeneutic-interpretative with open coding analysis, we observed two main themes in relation to daily activities performance: on one hand, the need of keep meaningful roles and on the other hand, the auto marginality and the dependence sensation imposed by the social environment. These findings highlight the importance of considering the perception of the person with dementia and their environment in relation to the possibilities of participation in daily activities, considering that not only the clinical symptoms may affect the participation, it may be affected also by the interpretation about daily situations.
\end{abstract}

\section{Keywords:}

Dementia; Activities of Daily Living; Perception; Task Performance.

Fecha de recepción: 09/09/2016

Fecha aceptación: 21/11/2016

1 Licenciado en Ciencias de la ocupación, Universidad de Chile. Magister en Envejecimiento y Calidad de vida, Instituto de Nutrición y Tecnología de los Alimentos (INTA), Universidad de Chile, Santiago, Chile. Escuela de Terapia Ocupacional, Universidad Bernardo O’Higgins, Santiago, Chile. Correo: jose.aravena@inta.uchile.cl

2 Terapeuta Ocupacional, Licenciada en Ciencias de la ocupación, Universidad de Chile. Centro Kintun, Peñalolén, Chile. Correo: dtello@penalolen.cl.

Terapeuta Ocupacional, Licenciada en Ciencia de la ocupación, Universidad Mayor. Centro Kintun, Peñalolén, Chile. Correo: tcallejas@penalolen.cl. 


\section{INTRODUCCIÓN}

El deterioro cognitivo y las demencias se caracterizan principalmente por ser cuadros asociados a fallas adquiridas en el rendimiento cognitivo (Blazer, 2013). Si bien existen diversas pruebas neuropsicológicas sensibles a la manifestación de algún déficit en algún área cognitiva, estas fallas habitualmente no se hacen evidentes hasta que comienzan a interrumpir el desempeño independiente y autónomo en las actividades de la vida diaria (Jekel et al., 2015)it is not clear if there are IADL domains that are consistently affected across patients with $\mathrm{MCl}$. In this systematic review, therefore, we aimed to summarize research results regarding the performance of $\mathrm{MCl}$ patients in specific IADL (sub. Las personas con deterioro cognitivo y demencia son altamente vulnerables a diversas situaciones que podrían afectar su salud (desnutrición, dependencia impuesta, estigma, negligencia en el cuidado, iatrogenia). Entre estas, una de las situaciones que se presentan de forma cotidiana en estos sujetos y sus cuidadores son los accidentes y situaciones de peligro en el hogar y la comunidad, lo que puede llevar a necesitar más cuidados y supervisión continua (Alzeimer's Association, 2015; Bourgeois, Couturier, \& Tyrrell, 2009; Härlein, Dassen, Halfens, \& Heinze, 2009).

Dentro de las dificultades en las actividades de la vida diaria que se presentan con mayor frecuencia en etapas de deterioro cognitivo leve y demencias iniciales son el recordar eventos o fechas importantes, concentrarse en la lectura, describir que cosas vio o escuchó recientemente, participar en una conversación, recibir y entregar recados, hacer dos cosas al mismo tiempo, afrontar situaciones desconocidas o poco familiares, realizar actividades de forma segura, y hacer una tarea bajo presión (Reppermund et al., 2011). Así mismo, se ha observado que la falla en la ejecución de diversas actividades de la vida diaria puede ser un predictor importante de deterioro cognitivo en la persona mayor. Hay estudios que mencionan que las primeras actividades de la vida diaria que pueden verse interrumpidas o entorpecidas en su ejecución, producto de un cuadro de deterioro cognitivo, son revisar cuentas por internet, pago de cuentas, manejo de medicamentos, uso de teléfono, reparar objetos pequeños, retención de información importante, realización de compras y manejo de finanzas, siendo estas últimas dos, las más sensibles al momento de evidenciar fallas producto de un cuadro de deterioro cognitivo leve o demencia (Burton,
Strauss, Bunce, Hunter, \& Hultsch, 2009; Rodakowski et al., 2014).

Pero no sólo los síntomas propios del cuadro podrían ser un factor que influye en el desempeño en actividades de la vida diaria por parte de la persona, sino que también el estigma asociado a la propia enfermedad (Burgener, et al, 2015). Producto del desconocimiento existente, generalmente las dificultades cognitivas o el diagnóstico de demencia se suele asociar a dependencia y necesidad de cuidados constantes, incluso en etapas leves donde la persona aún conserva una reserva funcional y una capacidad de insight para tomar decisiones (Zanetti, Geroldi, Frisoni, Bianchetti, \& Trabucchi, 1999). Esto podría llevar a situaciones de dependencia impuesta que limiten la participación de la persona con demencia (PcDem) en actividades que sean de importancia e interés para ella, y a su vez limitar la comprensión que tiene su entorno en relación a la problemática que tiene la persona (Burgener, Buckwalter, Perkhounkova, \& Liu, 2015).

Es en base a esto que el siguiente estudio busca conocer cuál es la percepción que tienen las personas con demencia leve participantes del Centro Kintun, en relación a su participación en actividades de la vida diaria. A partir de esta información se pretende identificar la percepción que tiene el grupo de personas mayores en relación a su desempeño diario en las actividades de la vida diaria, y que factores podrían influir en su sensación de competencia en la ejecución de actividades cotidianas.

\section{MÉTODO}

Estudio exploratorio de carácter cualitativo efectuado en el Centro de atención a personas con demencia "Kintun" de la comuna de Peñalolén, Santiago durante el mes de octubre del 2015. Se utilizó una metodología del tipo cualitativa con el fin de poder indagar y rescatar la percepción que tienen los sujetos en base a sus experiencias personales y pensamientos en relación al tema en cuestión, al momento de participar de este estudio (Minayo \& De, 2010).

El estudio se llevó a cabo en un grupo de 13 personas con cuadro compatible con demencia inicial o leve (Escala de deterioro global GDS Reisberg: estadio 4) (Reisberg, Ferris, De Leon, \& Crook, 1982), que viven en la comuna de Peñalolén junto a sus familias, y 
que son participantes del Centro Kitun en modalidad de una vez por semana (asisten al centro los días viernes de 10:00 am a 14:00 hrs). Durante su participación habitual en el Centro Kintun, los sujetos voluntariamente se hacen parte de una serie de actividades de carácter terapéutico dirigidas por profesionales de la salud en compañía de voluntarios (Terapeuta ocupacional, Kinesiólogo, técnico paramédico) durante un periodo aproximado de 6 meses (el cual puede variar según el caso), sumado a intervenciones individuales (visitas domiciliarias, evaluaciones, consejerías) para ellos y sus familias. Al momento de realizar este estudio, la mayoría de los participantes llevaba entre 4 a 8 meses de participación en el Centro Kintun.

\section{Recolección de información}

Con el fin de conocer cuál es la percepción que presentan las PcDem en relación al desempeño en la ejecución de sus actividades cotidianas, se realizó una recolección de información mediante la modalidad de grupo focal, formando grupos de 4 y 5 personas. El grupo focal permite que los participantes del estudio puedan dar sus opiniones en relación a diversos tópicos, generando reflexiones y respuestas desde los individuos y discursos como grupo interactivo de sujetos (Halcomb, Gholizadeh, DiGiacomo, Phillips, \& Davidson, 2007). Cada grupo contaba con un monitor (1 terapeuta ocupacional y 2 internos de terapia ocupacional) que dirigió de forma abierta el grupo focal en formato conversación, en relación a los siguientes tópicos:

- Actividades que son de alta importancia para ellos.

- $\quad$ Actividades que ellos percibían que les costaba más realizar.

Previo a los grupos focales, los entrevistadores prepararon la sesión y acordaron mutuamente como se conduciría la conversación y en qué puntos se haría énfasis. La duración de la actividad fue de 20 a $30 \mathrm{mi}$ nutos, y se efectuó de forma simultánea en una sala de actividades del Centro Kintun.

\section{Caracterización de los participantes}

Para la caracterización de los sujetos, se recolectó información socio-demográfica (edad, sexo, número de personas con las que vive), y características clíni- cas como la severidad de la demencia (evaluada con Escala de deterioro global GDS Reisberg) (Reisberg et al., 1982), y el nivel de independencia en actividades de la vida diaria reportado por el cuidador principal de la PcDem a través de la Escala Alzheimer's Disease Cooperative Study (ADCS-ADL) (Galasko et al., 1997).

Todas estas variables fueron recolectadas al ingreso del programa por parte de un equipo multidisciplinario (Médico geriatra, Psicóloga y Terapeuta ocupacional).

\section{Análisis de los resultados}

Se realizó una caracterización primaria de los participantes a través de estadísticos descriptivos (mediana, desviación estándar, mínimos y máximos) y frecuencias (considerando porcentajes) con el fin de conocer algunas características de los sujetos participantes del estudio. Los grupos focales se analizaron mediante un enfoque interpretativo-hermenéutico, generando categorías temáticas a través de un análisis de codificación abierta de las opiniones recolectadas y transcritas según los discursos emitidos por las personas participantes. Se seleccionaron frases textuales emitidas por los participantes que sirvieran para ilustrar y reforzar los resultados y componentes temáticos emergentes.

\section{Consideraciones éticas}

Todos los participantes en compañía de su cuidador o persona de referencia principal firmaron un consentimiento informado de participación para el programa Kintun y para la participación en este estudio. Este consentimiento informado fue leído y explicado de forma detallada para que la persona pudiera entenderlo. Antes de comenzar y al finalizar los grupos focales, se les volvió a explicar a los sujetos acerca de su rol e implicancias en la participación de este estudio, y se les preguntó si deseaban participar y entregar voluntariamente la información otorgada.

\section{Resultados}

En la tabla 1 se describen las características de las 13 personas entrevistadas. El promedio de edad fue de $77,5( \pm 5,35)$ años, el $69,2 \%$ (9 personas) correspondieron a mujeres, y donde todas las personas vivían a lo 
menos con un familiar (el 76,9\% vivía con dos o más personas en su hogar). Todos los sujetos entrevistados presentaban demencia leve, y el nivel de independencia en las AVD reportado por el cuidador fue de un promedio de 51,7 ( $\pm 7,71$; Mín.= 37 - Máx.=63) pts, ósea de acuerdo a los cuidadores todos los sujetos reportaban algún grado de dependencia en más de alguna actividad de la vida diaria.

Tabla 1.

Descripción de las personas con demencia participantes

\begin{tabular}{|c|c|c|c|c|c|}
\hline Persona & $\begin{array}{l}\text { Edad } \\
\text { (años) }\end{array}$ & Sexo & $\begin{array}{l}\text { Número de personas } \\
\text { con las que vive }\end{array}$ & $\begin{array}{c}\text { Severidad de la } \\
\text { demencia (GDS-R) }\end{array}$ & $\begin{array}{l}\text { Nivel de independencia en AVD } \\
\text { reportado por cuidador (ADCS-ADL) }\end{array}$ \\
\hline 1 & 77 & Hombre & 2 & Leve & $40 / 78$ pts. \\
\hline 2 & 78 & Hombre & 1 & Leve & $48 / 78$ pts. \\
\hline 3 & 67 & Mujer & 2 & Leve & 63 / 78 pts. \\
\hline 4 & 73 & Mujer & 5 & Leve & 47 / 78 pts. \\
\hline 5 & 79 & Mujer & 2 & Leve & $61 / 78$ pts. \\
\hline 6 & 84 & Mujer & 2 & Leve & 46 / 78 pts. \\
\hline 7 & 84 & Hombre & 3 & Leve & $54 / 78$ pts. \\
\hline 8 & 85 & Mujer & 4 & Leve & 55 / 78 pts. \\
\hline 9 & 79 & Mujer & 3 & Leve & 37 / 78 pts. \\
\hline 10 & 72 & Mujer & 1 & Leve & 58 / 78 pts. \\
\hline 11 & 78 & Mujer & 3 & Leve & 54 / 78 pts. \\
\hline 12 & 80 & Mujer & 1 & Leve & 54 / 78 pts. \\
\hline 13 & 72 & Hombre & 4 & Leve & 55 / 78 pts. \\
\hline $\begin{array}{c}\text { GDS-R: Escala de } \\
\text { deterioro global de } \\
\text { Resiberg. } \\
\text { ADCS-ADL: } \\
\text { Azheimer's Disease } \\
\text { Cooperative Study } \\
\text { - Activities of Daily } \\
\text { Living Scale. } \\
\text { Pts.: Puntos. }\end{array}$ & & & & & \\
\hline
\end{tabular}

De los grupos focales realizados, emergieron dos grandes temáticas que son relevantes cotidianamente para las PcDem participantes, ilustradas en la Figura 1:

\section{Mantención de actividades y roles relevantes.}

Las actividades más relevantes que las personas con demencia emitieron fueron aquellas que tenían algún sentido biográfico para ellos, es decir, actividades relacionadas con sus roles pasados. En el caso de las mujeres, era muy importante mantener la independencia para cocinar, hacer las tareas de limpieza y orden del hogar, y realizar compras. Y para todos los usuarios, las actividades que reportaban de mayor importancia era la realización de tareas del hogar, realizar compras, manejar el dinero y el salir a la comunidad. Así se evidencia en las siguientes frases: 
"Para mí lo más importante es mantener mi casita limpia y la comida echa para todos... Yo siempre me he encargado de eso en la casa, y mi hija lo sabe..."

"Hay que llevar la cuenta de la plata que uno tiene... sino uno no sabe lo que puede pasar... siempre es importante tener plata para comprarse las cosas que uno quiere... además si hay que ayudar con algo... es importante la familia..."

Si la continuidad de ese rol no se puede llevar a cabo de forma efectiva (por ejemplo, en el caso de una persona que se jubila y no puede seguir ejerciendo su rol de trabajador), las personas reportaban que trataban de adaptarse a las demandas y necesidades que ofrecía el ambiente. Esto se daba de forma más frecuente en el caso de los hombres, quienes referían mayores situaciones asociadas a la adaptación y búsqueda de un rol significativo dentro del hogar y núcleo familiar, a pesar de las barreras que podían encontrar.

Se observa que la continuidad en la ejecución de actividades biográficamente importantes es determinada por los espacios y oportunidades que ofrece el ambiente, y por el significado histórico que tiene para los sujetos. Esto se evidencia en la siguiente frase:

\begin{abstract}
"Yo trabajé toda mi vida... ahora soy jubilado y paso todo el día en la casa... trato de ayudarle a mi señora a ordenar... pero mi señora se enoja porque lo hago mal...entonces prefiero irme a mi taller $y$ arreglar alguna cosa de la casa... siempre hay algo que arreglar"
\end{abstract}

\section{Dependencia impuesta vs fallas cognitivas}

En relación a la necesidad de ayuda en la ejecución de actividades, se observó dos tipos de discursos. Por un lado, las personas que lograban percibir sus dificultades cognitivas, entendían y atribuían las fallas o limitaciones en la ejecución de las actividades de la vida diaria a sus propias fallas cognitivas. Esas personas comenzaban a marginarse voluntariamente de diversas actividades por temor a presentar alguna falla (ejemplo dejar de salir a la calle o comprar por miedo a extraviarse), tal como se ve puede observar en los discursos:
"A mí me encantaba salir a comprar y pasear sola... pero cuando empecé a darme cuenta de que me estaba fallando la memoria, empecé a quedarme en la casa... no quería salir... ahora me da miedo salir sola, porque me da miedo perderme...".

"Antes de los problemas de memoria yo guardaba toda mi plata... pero un día me di cuenta de que se me perdía a cada rato, así que ahora le paso toda mi plata a mi hija... ella tiene mi plata y me compra mis cosas... me da pena que mi hija tenga que encargarse de mi...".

Por otra parte, las personas que entendían las limitaciones como una dependencia impuesta por el entorno, no atribuían sus limitaciones funcionales a fallas cognitivas, sino que más bien a un exceso de sobreprotección del entorno que les limitaba poder seguir participando de actividades que para ellos eran significativas (ejemplo el poder seguir cocinando, o el salir a comprar). Esta idea se ilustra mediante las siguientes frases:

"Yo no entiendo a mi hija, no me deja hacer nada... yo agarro mis cosas para salir a comprar algo pa' la casa... y parece que me estuviera escapando porqué altiro me grita 'pa donde vay'... yo no soy na niña para que me vigilen así".

"Mijito, yo era la cocinera de esta casa... las mejores cazuelas las hacia yo... ahora no me dejan prender la cocina, no me dejan prender el calefón, no me dejan salir a comprar... pucha me siento inútil, no puedo estar ni en mi propia cocina... si la memoria no la tengo tan mala...".

En relación a la percepción de dependencia en actividades diarias reportadas por las personas mayores con demencia, esta es coherente con las observaciones entregadas por los cuidadores (a través del puntaje en la escala ADCS-ADL), quienes en todos los casos reportan algún grado de dependencia en las actividades diarias, en mayor o menor medida (Tabla 1). Sin embargo, de acuerdo a la percepción de las personas con demencia, esta dependencia no sólo tiene origen en las dificulta- 
des cognitivas, sino que también en la sobreprotección y dependencia impuesta por el entorno social.

Es relevante mencionar que en todos los casos los usuarios refieren como positivo el que se les entreguen herramientas y estrategias para mejorar su desempeño y a la vez que se le de educación a sus familias para evitar la sobreprotección.

Figura 1.

Principales temáticas emergentes de los grupos focales de personas con demencia.

\section{Mantención de actividades relevantes y significativas}

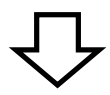

Continuidad de un rol e importancia biográfica

- Cocinar

- Tareas del hogar

- Compras

- Manejo de dinero

- Salir a la comunidad

Fuente: propia del autor.

\section{Dependencia impuesta v/s Fallas cognitivas

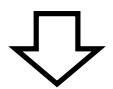

Sujetos con percepción de fallas cognitivas: atribución de las limitaciones a fallas personales. Auto-restricción en actividades.

Usuarios que perciben limitaciones a dependencia impuesta por el entorno: exceso de sobreprotección limita continuidad de actividades.

\section{Discusión}

Las personas con demencia presentan fallas en su autonomía y capacidad para realizar sus actividades de la vida diaria producto de los síntomas propios del cuadro clínico, lo que frecuentemente significa la necesidad de un tercero para resguardar la salud y calidad de vida. Sin embargo, no sólo situaciones asociadas a la sintomatología clínica del cuadro pueden causar una situación de dependencia, sino que también la propia percepción de autoeficacia que tiene el sujeto en relación a sus capacidades (Clare et al., 2012), y a su vez la dependencia impuesta por el entorno ya sea por los errores que cotidianamente comete o por las posibles fallas que podría presentar

En este estudio se pudo vislumbrar desde la percepción de la PcDem aspectos fundamentales relacionados a su desempeño cotidiano en las actividades de la vida diaria. Dichos componentes son la necesidad de mantener los roles y la participación en actividades que han sido históricamente importantes, y la exclusión en la participación en actividades, la cual según la información recolectada se basaba en la auto marginación producto de la conciencia de fallas cognitivas, o en la sensación de limitación y sobreprotección del entorno (Figura 1). Estos últimos sujetos no necesariamente percibían fallas en su desempeño, sino que más bien el ambiente era quien los limitaba.

Dichos hallazgos pueden ser interpretados desde la perspectiva de la interacción existente entre la persona, su ambiente y las ocupaciones que efectúa (Law et al., 1996). La relación que se establece entre la PcDem y su entorno físico y social es en base a las percepciones e interpretaciones que realizan los sujetos en relación a los eventos cotidianos (Figura 2) (Clare et al., 2012). Estos eventos cotidianos corresponden a las ocupaciones que realizan las personas, puesto que si el desempeño se sigue manteniendo de acuerdo a lo esperado, el ambiente y el sujeto no realizarán ajustes para mantener dicha relación; no así cuando el desempeño ocupacional presenta modificaciones (como es el caso de la persona con déficit cognitivo), en donde la persona modificará conductas si percibe que su desempeño esta alterado (ejemplo: la persona con demencia deja de realizar una actividad). Si el sujeto no logra percatarse de dichos cambios, el ambiente tratará de realizar ajustes 
para sopesar dichas modificaciones (ejemplo: aumentar la supervisión de la persona en sus actividades por parte de su ambiente social). Estos cambios del ambiente no deben ser clasificados en todos los casos como dependencia impuesta, puesto podría depender de como la persona con demencia interpreta dicha situación en relación a cómo percibe sus capacidades.

Resulta todo un reto la intervención y trabajo con personas que presentan cuadros de deterioro cognitivo o demencia en estadios iniciales o leves, puesto si bien presentan fallas cognitivas que afectan su desempeño funcional, siguen siendo personas que preservan un nivel de autonomía y dependencia que les permite seguir participando en actividades en su hogar y en la comu- nidad, y que se identifican con los diversos roles que han indo construyendo a lo largo de su biografía (Clare et al., 2013). Es aquí donde muchas veces se genera un dilema importante para la familia de las personas con deterioro cognitivo, puesto al evidenciar las fallas que comienzan a presentar en las actividades cotidianas muchas veces terminan generando una dependencia impuesta con el fin de resguardar la seguridad de la persona, o por el contrario al evidenciar que aún pueden realizar actividades existe una escaza supervisión de la ejecución de las actividades diarias, lo que en muchas ocasiones puede ocasionar situaciones de peligro (Horvath et al., 2005).

Figura 2.

Interacción entre la persona con demencia y su ambiente físico y social durante su desempeño cotidiano

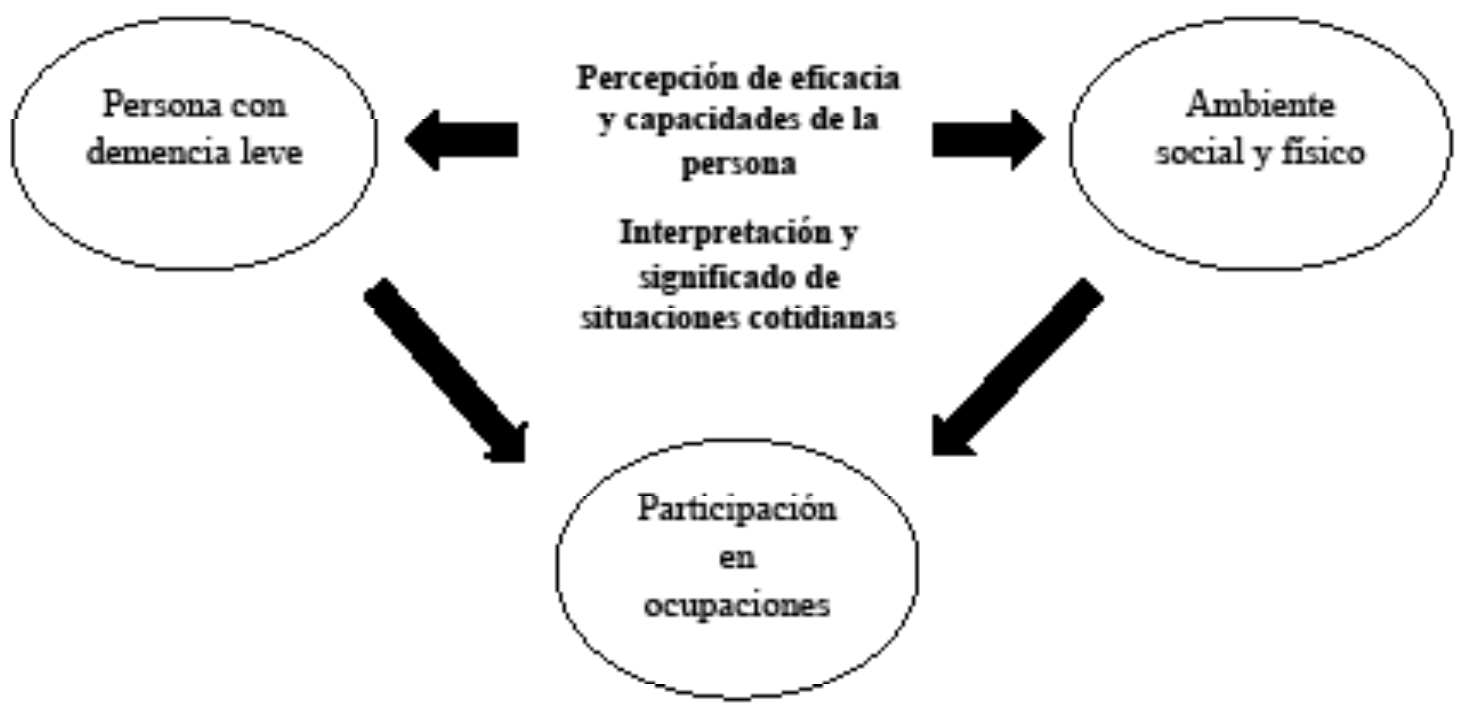

Figura: propia del autor.

Este estudio aporta entregando una nueva mirada en relación a la independencia y dependencia en actividades de la vida diaria en personas mayores con demencia leve, lo que es un aporte para la práctica de la Terapia ocupacional en esta área, puesto favorece la inclusión de nuevos elementos para mejorar el abordaje terapéutico de este tipo de usuarios y sus entornos. En lo concreto, se propone cómo fundamental que todo abordaje de Terapia ocupacional en personas con demencia leve deba considerar la biografía e historia ocu- pacional como elementos centrales para la promoción de la participación y la independencia en actividades cotidianas. Por otra parte, el considerar la percpeción de eficacia y de desempeño del sujeto puede ser un elemento trascendental para lograr nuevos beneficios en la intervención, incorporando beneficios que van más allá de la preservación de la capacidad funcional del sujeto, sino que también apuntan a la reducción del estigma y auto-estigma, incremento en la sensación de eficacia y placer en actividades, reducción de síntomas 
depresivos, y mejoría en la percpeción de calidad de vida y bienestar.

Bajo esta mirada se propone que los Terapeutas ocupacionales, además de considerar los síntomas y manifestación clínica del cuadro para sus intervenciones en PcDem y sus familias, también deben incorporar el significado e interpretación que cada uno de los sujetos les entrega a las situaciones enmarcadas en actividades de la vida diaria. A partir de esto podrán diseñarse tratamientos que tengan una mejor adherencia y que respondan de forma más oportuna a las necesidades y problemáticas percibidas por estos sujetos y su entorno.

Modelos como el transaccional de autonomía en PcDem (Gajardo \& Cifuentes, 2014), pueden ser una propuesta teórica de gran aporte para respaldar y darle racionalidad a la intervención de Terapia ocupacional en las demencias, puesto en concordancia con los hallazgos de este estudio, existe una necesidad de ajuste cotidiano (por parte de la PcDem y de su entorno) de las situaciones ocupacionales que viven los sujetos, a las demandas, capacidades y expectativas de participación que tienen los diversos actores involucrados.

\section{REFERENCIAS BIBLIOGRÁFICAS}

Alzeimer's Association. (2015). Staying safe: Steps to take for a person with dementia. Alzheimer's Association. https://doi. org/10.4135/9781446288672.n6

Blazer, D. (2013). Neurocognitive disorders in DSM-5. The American Journal of Psychiatry, 170(6), 585-7. https://doi.org/10.1176/appi. ajp.2013.13020179

Bourgeois, J., Couturier, P., \& Tyrrell, J. (2009). Safety at home for people with dementia: Preliminary evaluation of situations-at-risk in a French geriatric memory clinic. Psychol NeuroPsychiatr Vieil, 7(3), 213-224.

Clare, L., Whitaker, C. J., Nelis, S. M., Martyr, A., Markova, I. S., Roth, I., ... Morris, R. G. (2013). Self-concept in early stage dementia: Profile, course, correlates, predictors and implications for quality of life. International Journal of Geriatric Psychiatry, 28(5), 494-503. https://doi.org/10.1002/gps.3852

Gajardo, J., \& Cifuentes, D. F. (2014). Un modelo transaccional o contextualizado de autonomía en personas con demencia. Revista Chilena de Terapia Ocupacional, 14(2), Pag. 185-195. Retrieved from http://www.revistaterapiaocupacional.uchile.cl/index.php/RTO/ article/view/35721

Galasko, D., Bennett, D., Sano, M., Ernesto, C., Thomas, R., Grudman, M., \& Ferris, S. (1997). An inventory to assess activities of daily living for clinical trials in Alzheimer's Disease. Alzheimer Disease \& Associated Disorders, 11 (Suppl 2), S33-9.
Halcomb, E. J., Gholizadeh, L., DiGiacomo, M., Phillips, J., \& Davidson, P. M. (2007). Literature review: Considerations in undertaking focus group research with culturally and linguistically diverse groups. Journal of Clinical Nursing, 16(6), 1000-1011. https://doi. org/10.1111/j.1365-2702.2006.01760.x

Härlein, J., Dassen, T., Halfens, R. J. G., \& Heinze, C. (2009). Fall risk factors in older people with dementia or cognitive impairment: a systematic review. Journal of Advanced Nursing, 65(5), 922-33. https://doi.org/10.1111/j.1365-2648.2008.04950.x

Horvath, K. J., Hurley, A. C., Duffy, M. E., Gauthier, M. A., Harvey, R. M., Trudeau, S. a, ... Smith, S. J. (2005). Caregiver competence to prevent home injury to the care recipient with dementia. Rehabilitation Nursing: The Official Journal of the Association of Rehabilitation Nurses, 30(5), 189-196; discussion 197. https://doi. org/10.1002/j.2048-7940.2005.tb00109.x

Jekel, K., Damian, M., Wattmo, C., Hausner, L., Bullock, R., Connelly, P. J., ... Frölich, L. (2015). Mild cognitive impairment and deficits in instrumental activities of daily living: a systematic review. Alzheimer's Research \& Therapy, 7(1), 17. https://doi.org/10.1186/ s13195-015-0099-0

Law, M., Cooper, B., Strong, S., Stewart, D., Rigby, P., \& Letts, L. (1996). The Person-Environment-Occupational model: A transactive approach to occupational performance. Canadian Journal of Occupational Therapy, 63(1), 219-233.

Minayo, S., \& De, M. C. (2010). Los conceptos estructurantes de la investigación cualitativa. Salud Colectiva, 6(3), 251-261.

Reisberg, B., Ferris, S. H., De Leon, M. J., \& Crook, T. (1982). The global deterioration scale for assessment of primary degenerative dementia. American Journal of Psychiatry, 139(9), 1136-1139.

Reppermund, S., Sachdev, P. S., Crawford, J., Kochan, N. A., Slavin, M. J., Kang, K., ... Brodaty, H. (2011). The relationship of neuropsychological function to instrumental activities of daily living in mild cognitive impairment. International Journal of Geriatric Psychiatry, 26(8), 843-852. https://doi.org/10.1002/gps.2612

Rodakowski, J., Skidmore, E. R., Reynolds, C. F., Dew, M. A., Butters, M. a, Holm, M. B., ... Rogers, J. C. (2014). Can Performance on Daily Activities Discriminate Between Older Adults with Normal Cognitive Function and Those with Mild Cognitive Impairment? Journal of the American Geriatrics Society, 1347-1352. https://doi. org/10.1111/jgs.12878

Zanetti, O., Geroldi, C., Frisoni, G. B., Bianchetti, A., \& Trabucchi, M. (1999). Contrasting results between caregiver's report and direct assessment of activities of daily living in patients affected by mild and very mild dementia: the contribution of the caregiver's personal characteristics. Journal of the American Geriatrics Society, 47(2), 196-202. https://doi.org/10.1111/j.1532-5415.1999.tb04578.x 